\title{
An L-type substellar object in Orion: reaching the mass boundary between brown dwarfs and giant planets
}

\author{
M. R. Zapatero Osorio, V. J. S. Béjar, R. Rebolo ${ }^{1}$ \\ Instituto de Astrofísica de Canarias, E-38200 La Laguna, Tenerife, Spain \\ E. L. Martín, and G. Basri \\ Astronomy Department, University of California, Berkeley, CA 94720, USA \\ e-mail addresses: mosorio@ll.iac.es, vbejar@ll.iac.es, rrl@ll.iac.es \\ ege@popsicle.berkeley.edu, basri@soleil.berkeley.edu
}

\begin{abstract}
We present near-infrared photometry ( $J$-band) and low-resolution optical spectroscopy $(600-1000 \mathrm{~nm})$ for one of the faintest substellar member candidates in the young $\sigma$ Orionis cluster, SOri $47(I=20.53$, Béjar et al. 1999). Its very red $(I-J)=3.3 \pm 0.1$ color and its optical spectrum allow us to classify SOri 47 as an L1.5-type object which fits the low-luminosity end of the cluster photometric and spectroscopic sequences. It also displays atmospheric features indicative of low gravity such as weak alkaline lines and hydride and oxide bands, consistent with the expectation for a very young object still undergoing gravitational collapse. Our data lead us to conclude that S Ori 47 is a true substellar member of the $\sigma$ Orionis cluster. Additionally, we present the detection of Li I in its atmosphere which provides an independent confirmation of youth and substellarity. Using current theoretical evolutionary tracks and adopting an age interval of $1-5 \mathrm{Myr}$ for the $\sigma$ Orionis cluster, we estimate the mass of S Ori 47 at $0.015 \pm 0.005 M_{\odot}$, i.e. at the minimum mass for deuterium burning, which has been proposed as a definition for the boundary between brown dwarfs and giant planets. S Ori 47 could well be the result of a natural extension of the process of cloud fragmentation down to the deuterium burning mass limit; a less likely alternative is that it has originated from a protoplanetary disc around a more massive cluster member and later ejected from its orbit due to interacting effects within this rather sparse $\left(\sim 12\right.$ objects $\left.\mathrm{pc}^{-3}\right)$ young cluster. The study of this object serves as a guide for future deep searches for free-floating objects with planetary masses.
\end{abstract}

Subject headings: open clusters and associations: individual ( $\sigma$ Orionis) - stars: evolution - stars: low-mass, brown dwarfs

${ }^{1}$ Consejo Superior de Investigaciones Científicas, CSIC. Spain 


\section{Introduction}

Nearby, young clusters offer unique opportunities to establish the observational properties of objects at and below the hydrogen burning limit (brown dwarfs, BDs), and constitute an ideal scenario to deepen in our knowledge of the initial mass function (IMF) in the substellar regime. The advantage of searching in these clusters is that important parameters like age, distance, and metallicity are relatively well known, and thus, they can be used for constraining masses and luminosities of cluster members. The first claims of the discovery of BDs in the Pleiades (Rebolo, Zapatero Osorio \& Martín 1995; Basri, Marcy \& Graham 1996) have been followed by recent deeper searches which considerably extend the area surveyed in this cluster (Bouvier et al. 1998; Stauffer et al. 1998; Zapatero Osorio et al. 1999) as well as in other young nearby clusters like Praesepe (Magazzù et al. 1998), Hyades (Gizis, Reid \& Monet 1999), $\rho$ Oph (Comeron et al. 1998), Chamaleon I (Comeron, Rieke, \& Neuhäuser 1999), and IC 348 (Luhman et al. 1998, 1999). These efforts were devoted to determine how far the IMF extends into the substellar domain. To date, BDs with masses of only $0.035 M_{\odot}$ have been confirmed in the Pleiades (Martín et al. 1998), and down to roughly $0.025 M_{\odot}$ in a few younger clusters (Luhman, Liebert \& Rieke 1997; Martín, Basri \& Zapatero Osorio 1999a; Béjar, Zapatero Osorio \& Rebolo 1999 ).

Very recently, Béjar et al. (1999; hereafter referred to as BOR99) have performed a deep $R I Z$ survey in the young $\sigma$ Orionis cluster (Walter et al. 1997), located within the Orion $1 \mathrm{~b}$ association. This work reveals a rich population of very low-mass stars and BDs in the cluster with short ages in the interval 1-5 Myr. About 40 substellar candidate members were found down to $I=21 \mathrm{mag}$. We present here the follow-up IR photometry and optical spectroscopy of a $\sigma$ Orionis faint candidate, S Ori 47 , which was se- lected for its very red $I-Z$ color. We have estimated a mass of $0.015 M_{\odot}$ for this object, confirming it as the least massive free-floating object discovered to date in the Orion star-forming region.

\section{Observations and Analysis}

Near-infrared observations ( $J$-band) of the substellar candidate S Ori 47 were performed in 1998 Dec. 12 and in 1999 Feb. 24 at the $1.5 \mathrm{~m}$ Carlos Sánchez Telescope (TCS, Teide Observatory on the Island of Tenerife) using the CIR infrared camera equipped with an $\mathrm{HgCdTe} 256 \times 256$ array. This detector provided $1^{\prime \prime}$ pixels and a field of view of about $4^{\prime} \times 4^{\prime}$. The total integration time per night was $1080 \mathrm{~s}$, the final image being the co-addition of twelve dithered exposures of $90 \mathrm{~s}$ each. Raw data were processed using standard techniques within the IRAF $\mathrm{F}^{2}$ environment. Instrumental aperture magnitudes were transformed into the UKIRT system by observing faint standard stars (Casali \& Hawarden 1992) at different airmasses. The photometric calibration of both nights showed a dispersion of $\pm 0.2 \mathrm{mag}$. We can give a smaller error bar to the measurement of S Ori 47 because close to it (at $\sim 42^{\prime \prime} \mathrm{E}, 10^{\prime \prime} \mathrm{S}$ ) and within the field of view of our images there is another cluster BD candidate (S Ori 27, M7 spectral type, BOR99) for which there is IR data of high precision (Béjar et al. 2000); S Ori 27 does not appear to be variable. We have also performed differential photometry of our target with respect to this brighter object. Table 1 summarizes the optical and the averaged $J$ photometry of S Ori 47 and Fig. 11 shows its location in the $I$ versus $I-J$ color-magnitude diagram.

Low-resolution spectroscopy was collected with the Keck II Low Resolution Imaging Spectrograph (Oke et al. 1995) in 1998 Dec. 21 us-

\footnotetext{
${ }^{2}$ IRAF is distributed by National Optical Astronomy Observatory, which is operated by the Association of Universities for Research in Astronomy, Inc., under contract with the National Science Foundation.
} 
ing the longslit mode. At the beginning of the exposure we rotated the slit until parallactic angle in order to minimize light losses due to refraction. We used a slit width projection onto the detector of 4 pixels $\left(1^{\prime \prime} .2\right)$ and the $150 \mathrm{~g} / \mathrm{mm}$ grating blazed at $750 \mathrm{~nm}$, which gave a nominal dispersion of $4.8 \AA /$ pix. One single exposure of $1800 \mathrm{~s}$ was taken. The spectrum was debiased, flat-fielded, optimal extracted and wavelength calibrated using the emission spectra of $\mathrm{HgNeAr}$ lamps. All these procedures were performed within IRAF. The instrumental signature of the observed data was removed by matching the spectrum of BRI0021-0214 obtained with the same instrumental configuration during that night with a previous one that we already had flux calibrated (Martín, Rebolo \& Zapatero Osorio 1996). Figure 2 (upper panel) displays the final spectrum of S Ori 47 (resolution $\sim 20 \AA$ ) where some features have been indicated as in Kirkpatrick et al. (1999).

The colors and optical spectrum of S Ori 47 are indicative of a cool object belonging to the recently proposed L spectral class (Martín et al. 1997). Molecular absorption band heads of $\mathrm{TiO}$ in the range 640-740 $\mathrm{nm}$ appear very weak in our spectrum while the hydrides $\mathrm{CaH}, \mathrm{CrH}$ and $\mathrm{FeH}$ are as strong as VO. We have used the classification schemes proposed by Kirkpatrick et al. (1999) and Martín et al. (1999b) based on several atomic, molecular and pseudocontinuum indices to determine an L1.5-type for S Ori 47 with a dispersion of half a subclass. Its optical spectrum shows great similarities with other young BDs of similar spectral types like G 196-3B (Rebolo et al. 1998) and Roque 25 (Martín et al. 1998). The strengths of the $\mathrm{CrH}$ and $\mathrm{FeH}$ bands and the Na I, Cs I and K I lines are lower in S Ori 47 relative to other L1-L2 type objects in the field discovered by the all-sky IR survey Denis (Delfosse et al. 1997; Martín et al. 1999b). We attribute this effect to low gravity of S Ori 47 demonstrating its youth. Its pre-main sequence nature is also inferred from the location of this object in
Fig. 1. S Ori 47 clearly lies above the photometric sequence of field dwarfs and nicely fits the extrapolation toward low luminosities of the $\sigma$ Orionis cluster optical/IR sequences. There are photometric and spectroscopic evidences for low reddening in the region where SOri 47 was found $\left(A_{V} \leq 0.1\right.$, BOR99). We note that the pattern of the cluster members in the $I$ versus $I-J$ diagram is well reproduced by the models (Burrows et al. 1997 and Baraffe et al. 1998) overplotted to the observations. The NextGen models by Baraffe et al. provide magnitudes and colors directly; we have used their bolometric corrections for an age of $5 \mathrm{Myr}$ in order to convert luminosities given by Burrows et al. into magnitudes. The spectroscopy and photometry of S Ori 47 support its membership in the $\sigma$ Orionis cluster. Because of its cool temperature and low luminosity the substellarity of this object is thus guaranteed.

An independent consistency check of the substellar nature of SOri 47 is given by the detection of lithium in its atmosphere. Figure 2 (lower panel) shows an enlargement around the Li I $670.8 \mathrm{~nm}$ line of the low-resolution spectrum of S Ori 47 in comparison to two other L1-L2 BDs in the field, Kelu 1 (Ruiz, Leggett \& Allard 1997) and G 196-3B. The spectra were taken with identical instrumental configuration and during the same observing night. However, the signal-tonoise ratio of S Ori 47, which is the faintest of the three objects, is lower at these wavelenghts and we can only claim a detection at $2-3 \sigma$ confidence level. The EW we measure is given in Table 1 . The presence of lithium in such a cool object is enough to confirm its substellar nature with a mass $\leq 0.05 M_{\odot}$ (Baraffe et al. 1998; D'Antona $\&$ Mazzitelli 1994). However, the evidence of its cluster membership from the color-magnitude diagram and spectral features will provide a better constraint on the mass and luminosity of S Ori 47.

$\mathrm{H} \alpha$ is not observed in strong emission (upper limit of $6 \AA$ ) at the resolution of S Ori 47's spectrum in Fig. 2. This is not unusual for young ob- 
jects with very cool temperatures. Among field L-dwarfs only few cases are known with $\mathrm{H} \alpha$ in emission, and typically EWs are below $4 \AA$ (Tinney et al. 1997; Kirkpatrick et al. 1999; Martín et al. 1999b). The chromospheric activity in the $\sigma$ Orionis cluster seems to diminish with decreasing mass along the cluster sequence. M-type BDs in the cluster show indications of considerable activity (BOR99); S Ori 47 with a much later class does not present evidences of an active chromosphere. This behaviour is also observed among Pleiades very low-mass members (Zapatero Osorio et al. 1997; Martín et al. 1998).

\section{Discussion and Final Remarks}

We have estimated the probability that S Ori 47 could be a contaminating field object (and not a member of $\sigma$ Orionis) using the number density $\left(0.01 \mathrm{pc}^{-3}\right)$ of early-L type objects found in the field (Delfosse et al. 1999; Reid et al. 1999). Adopting an absolute $\mathrm{M}_{I}$ magnitude of 15.1 mag for an L1-L2-type dwarf and considering the completeness limit and covered area of BOR99's survey, we obtain an extremely low contamination $(<1 \%)$. The discovery of S Ori 47 can be understood in terms of the much higher stellar/substellar density in the Orion region than in the field. On the basis of our optical/IR photometry and low-resolution spectroscopy we can safely conclude that S Ori 47 is a member of the cluster.

Several authors have addressed the temperature scale for L-dwarfs (Leggett, Allard \& Hauschildt 1998; Basri et al. 1999; Pavlenko, Zapatero Osorio \& Rebolo 1999), and they all suggest on a value of $2000 \pm 100 \mathrm{~K}$ for an L2-type object. However, this is a calibration done for field dwarfs with gravities larger than that of S Ori 47. Because the $T_{\text {eff }}$ scale for cool, low-gravity objects is several hundred degrees warmer (Luhman et al. 1997), an upward correction of 100-200 K may be required for S Ori 47, which is consistent with the predictions by evolutionary models. The luminosity of this object can be ob- tained from the Hipparcos distance $(352 \mathrm{pc})$ to the star $\sigma$ Orionis, which belongs to the cluster of the same name, and using the $J$ bolometric correction. We have estimated the latter from the Lyon "dusty" models (I. Baraffe 1999, private communication) as a function of the $I-J$ color, resulting $\mathrm{BC}_{J}=2.17$. This yields a luminosity for S Ori 47 of $\log L / L_{\odot}=-2.75 \pm 0.15$ dex.

Figure 3 displays the luminosity evolution of objects of different masses according to the recent evolutionary models of Burrows et al. (1997) and the Lyon "dusty" models . S Ori 47 is indicated in this diagram with a box that takes into account the error bar in luminosity and the age interval adopted for the $\sigma$ Orionis cluster (1-5 Myr with a most probable age of $3 \mathrm{Myr}$, BOR99). The mass of S Ori 47 can be derived from this figure at $0.015 \pm 0.005 M_{\odot}$, where the error bar comes from uncertainties in age and other observable parameters. We note that the models used here and those by D'Antona \& Mazzitelli (1997) predict very similar luminosities for young, low-mass objects, and therefore our result does not change significantly from model to model. However, we shall caution that the realibility of this mass estimate relies on the evolutionary tracks, and thus, it is subject to possible systematic effects arising from the description of atmospheres. Nevertheless, in relative terms we can safely conclude that S Ori 47 is the least massive substellar object so far identified in the Orion region, and moreover, it is the least massive L-type substellar object for which photometry and spectroscpy have allowed a mass determination with some precision.

The mass of S Ori 47 is remarkably close to the deuterium burning mass limit (DBL, 0.013$0.015 M_{\odot}$, Saumon et al. 1996; D'Antona \& Mazzitelli 1997). It has been proposed recently that planets should be defined as those substellar objects that never fuse deuterium, while BDs do it efficiently for some portion of their evolution (see Oppenheimer, Kulkarni \& Stauffer 1999, and references therein). The error bars in the mass determination of S Ori 47 can effectively locate 
it on either side of the DBL, so at this stage we cannot argue whether it is a very low-mass BD which will eventually deplete its initial deuterium content or a free-floating massive, giant planet that will preserve deuterium for its whole life. S Ori 47 provides evidence that objects with masses around the DBL can form in Nature. The fact that more photometric candidates with similar magnitudes in BOR99's survey still wait for membership confirmation indicate that the population of objects near $0.015 M_{\odot}$ may be rather numerous in Orion. If the $\sigma$ Orionis cluster IMF (Béjar et al. 2000) is representative of the field then old counterparts of these substellar bodies might be quite common in the galactic disk, and we would expect several tens populating the solar neighborhood $(d \leq 10 \mathrm{pc})$. At ages close to the Solar System their luminosities will have decreased by 4 orders of magnitude becoming extremely faint at all wavelengths, and their atmospheres will have cooled down to temperatures similar to those of Jupiter and Saturn, so it is expected their energy distributions resemble those of these planets. Objects with $\sim 0.01 M_{\odot}$ have been found at very close orbits around stars (Marcy \& Butler 1998, and references therein); their likely formation in a protoplanetary disk could be related to mass accretion onto a rocky core or to a gravitational collapse, or both. We cannot discard any of them as a possible origin for S Ori 47, which may have been born orbiting a more massive cluster member. Because of its low mass S Ori 47 might have experienced severe orbital disruptions which ejected it from its birth place. However, given the young age of the $\sigma$ Orionis cluster and its density $\left(n \sim 12\right.$ objects pc ${ }^{-3}$ ), this is not very likely because the number of encounters among the cluster stellar population is expected to be rather small (Laughlin \& Adams 1998; Fuente Marcos \& Fuente Marcos 1999). S Ori 47 could well have originated as a free-floating substellar cluster member, thus representing the natural extension of the IMF of objects formed via the pro- cess of cloud fragmentation.

We are indebted to I. Baraffe and the Lyon group for providing theoretical evolutionary tracks prior to publication. We also thank A. Burrows for providing an electronic version of his models. We are grateful to D. Ardila for his cooperation in obtaining some of the data. Data presented here were obtained at the W. M. Keck Observatory, which is operated as a scientific partnership among CALTECH, the University of California and NASA; and at the TCS telescope operated on the island of Tenerife in the Spanish Observatorio del Teide of the IAC. Partial finantial support was provided by the Spanish DGES project PB95-1132-C02-01. E.L.M. was partially supported by a fellowship of the Spanish Ministry of Education. G.B. acknowledges the support of NSF through grant AST96-18439. 


\section{REFERENCES}

Baraffe, I., Chabrier, G., Allard, F., \& Hauschildt, P. 1998, A\&A, 337, 403

Basri, G., Marcy, G. W., \& Graham, J. R. 1996, ApJ, 458, 600

Basri, G., et al. 1999, ApJ, submitted

Béjar, V. J. S., Zapatero Osorio, M. R., \& Rebolo, R. 1999, ApJ, 521, 671 (BOR99)

Béjar, V. J. S., et al. 2000, in preparation

Bouvier, J., Stauffer, J. R., Martín, E. L., Barrado y Navascués, D., Wallace, B., \& Béjar, V. J. S. 1998, A\&A, 336, 490

Burrows, A., Marley, M., Hubbard, W. B., et al. 1997, ApJ, 491, 856

Casali, M. M., \& Hawarden, T. G. 1992, JCMTUKIRT Newslt., 4, 33

Comeron, F., Rieke, G. H., Claes, P., Torra, J., \& Laureijs, R. J. 1998, A\&A, 335, 522

Comeron, F., Rieke, G. H., \& Neuhäuser, R. 1999, A\&A, 343, 477

D'Antona, F., \& Mazzitelli, I. 1994, ApJS, 90, 467

D'Antona, F., \& Mazzitelli, I. 1997, Mem. Soc. Astron. Italiana, 68, 807

Delfosse, X., Tinney, C. G., Forveille, T., et al. 1997, A\&A, 327, L25

Delfosse, X., Tinney, C. G., Forveille, T., et al. 1999, A\&AS, 135, 41

Fuente Marcos, C., \& Fuente Marcos, R. 1999, New. Astron., 4, 21

Gizis, J. E., Reid, I. N., \& Monet, D. G. 1999, AJ, in press

Kirkpatrick, J. D., Reid, I. N., Liebert, J., et al. 1999, ApJ, 519, 802
Laughlin, G., \& Adams, F. C. 1998, ApJ, 508, L171

Leggett, S. K., Allard, F., \& Hauschildt, P. H. 1998, ApJ, 509, 836

Luhman, K. 1999, ApJ, in press

Luhman, K., Liebert, J., \& Rieke, G. H. 1997, ApJ, 489, L165

Luhman, K., Rieke, G. H., Lada, C. J., \& Lada, E. A. 1998, ApJ, 508, 347

Magazzù, A., Rebolo, R., Zapatero Osorio, M. R., Martín, E. L., \& Hodgkin, S. T. 1998, ApJ, 497, L47

Marcy, G. W., \& Butler, R. P. 1998, ARA\&A, 36,57

Martín, E. L., Basri, G., Delfosse, X., \& Forveille, T. 1997, A\&A, 327, L29

Martín, E. L., Basri, G., \& Zapatero Osorio, M. R. 1999a, AJ, in press

Martín, E. L., Basri, G., Zapatero Osorio, M. R., Rebolo, R., \& García López, R. J. 1998, ApJ, 507, L41

Martín, E. L., Delfosse, X., Basri, G., Goldman, B., Forveille, T., \& Zapatero Osorio, M. R. 1999b, AJ, in press

Martín, E. L., Rebolo, R., \& Zapatero Osorio, M. R. 1996, ApJ, 469, 706

Oke, J. B., et al. 1995, PASP, 107, 375

Oppenheimer, B. R., Kulkarni, S. R., \& Stauffer, J. R. 1999, in Protostars and Planets IV, V. Mannings, A. Boss, S. Russell, eds. (Tucson: University of Arizona Press), in press

Pavlenko, Ya., Zapatero Osorio, M. R., \& Rebolo, R. 1999, A\&A, submitted

Rebolo, R., Zapatero Osorio, M. R., Madruga, S., Béjar, V. J. S., Arribas, S., \& Licandro, J. 1998, Science, 282, 1309 
Rebolo, R., Zapatero Osorio, M. R., \& Martín, E. L. 1995, Nature, 377, 129

Reid, I. N., Kirkpatrick, J. D., Liebert, J., et al. 1999, ApJ, 521, 613

Ruiz, M. T., Leggett, S. K., \& Allard, F. 1997, ApJ, 491, L107

Saumon, D, Hubbard, W. B., Burrows, A., Guillot, T., Lunine, J. I., \& Chabrier, G. 1996, ApJ, 460, 993

Stauffer, J. R., Schild, R., Barrado y Navascués, D., et al. 1998, ApJ, 504, 805

Tinney, C. G., Delfosse, X., \& Forveille, T., 1997, ApJ, 490, L95

Walter, F. M., Wolk, S. J., Freyberg, M., \& Schmitt, J. H. M. M. 1997, Mem. Soc. Astron. Italiana, 68, 1081

Zapatero Osorio, M. R., Rebolo, R., Martín, E. L., et al. 1997, ApJ, 491, L81

Zapatero Osorio, M. R., Rebolo, R., Martín, E. L., Hodgkin, S. T., Cossburn, M. R., Magazzù, A., Steele, I. A., \& Jameson, R. F. 1999, A\&AS, 134, 537

This 2-column preprint was prepared with the AAS LATEX macros $\mathrm{v} 4.0$. 


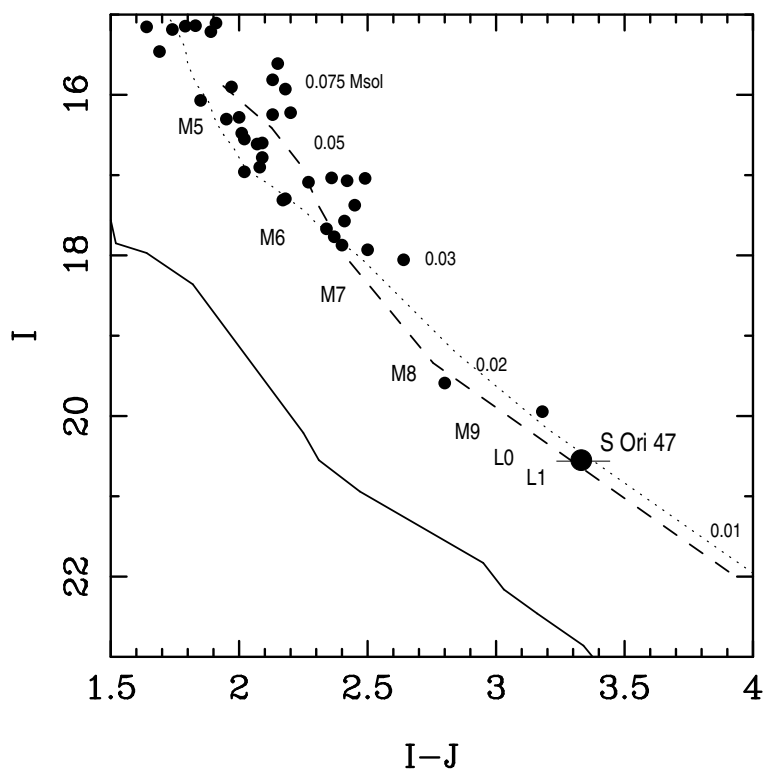

Fig. 1. - Location of S Ori 47 (large-size circle) in the optical-IR color-magnitude diagram. Some other $\sigma$ Orionis substellar cluster members (small-size circles, Béjar et al. 2000) are also plotted. The solid line corresponds to the average sequence of very low-mass dwarfs shifted to the cluster distance. Theoretical isochrones of Burrows et al. (1997, $3 \mathrm{Myr}$, dashed line) and Baraffe et al. (1998, NextGen 5 Myr, dotted line) are oveplotted to the data. Masses are given in solar units and spectral types are labeled as a function of the $I-J$ color.
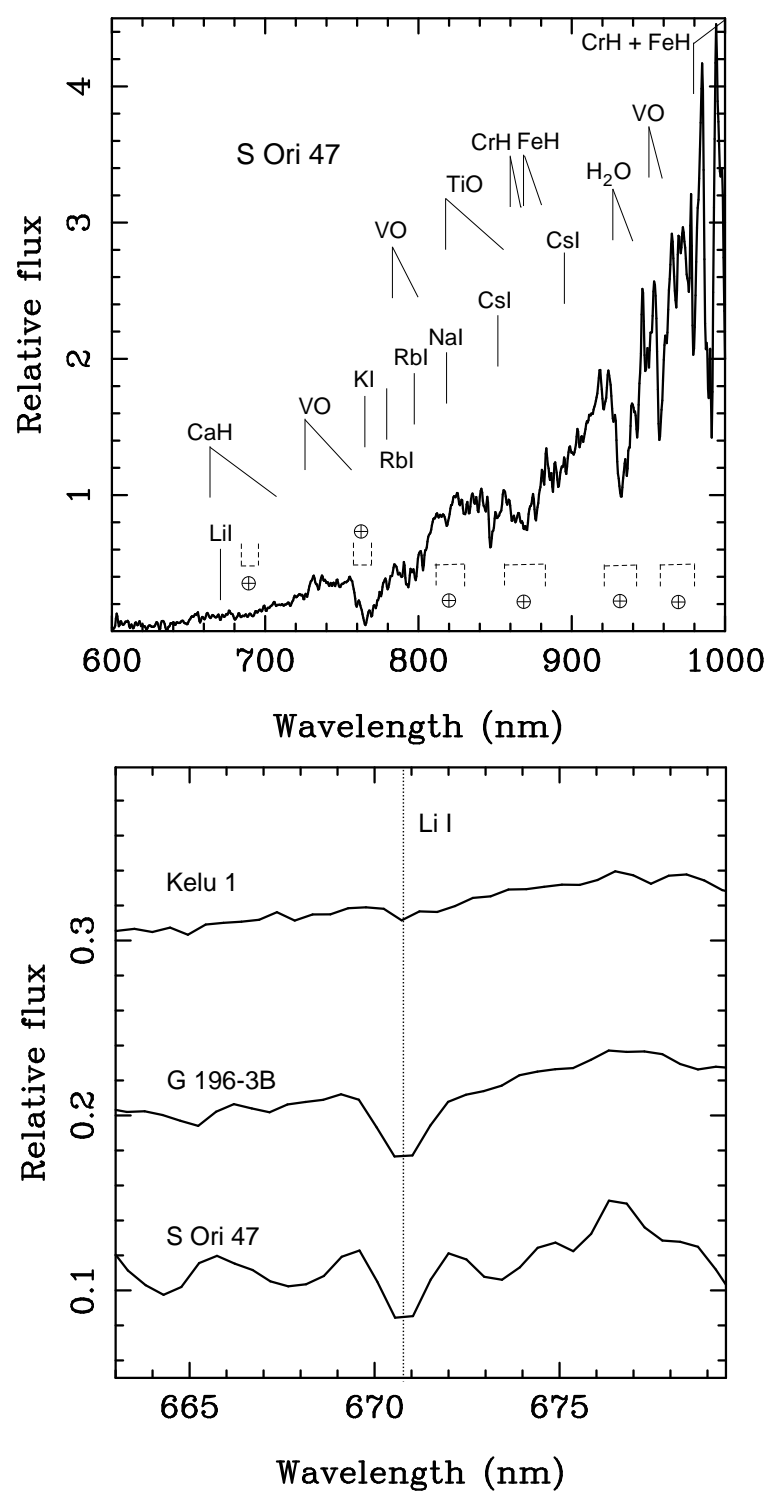

Fig. 2.- (Upper panel) Keck low-resolution spectrum of S Ori 47. It has been normalized at around $833.5 \mathrm{~nm}$. A boxcar smoothing of 3 pixels has been applied. Identification of some atomic and molecular features is given in the top. Telluric bands are marked with dashed lines. (Lower panel) Enlargement of S Ori 47's optical spectrum around the Li I resonance doublet. Two other L1-L2 brown dwarfs in the field observed with identical instrumental configuration (displaced by 0.1 units each) are shown for comparison. 


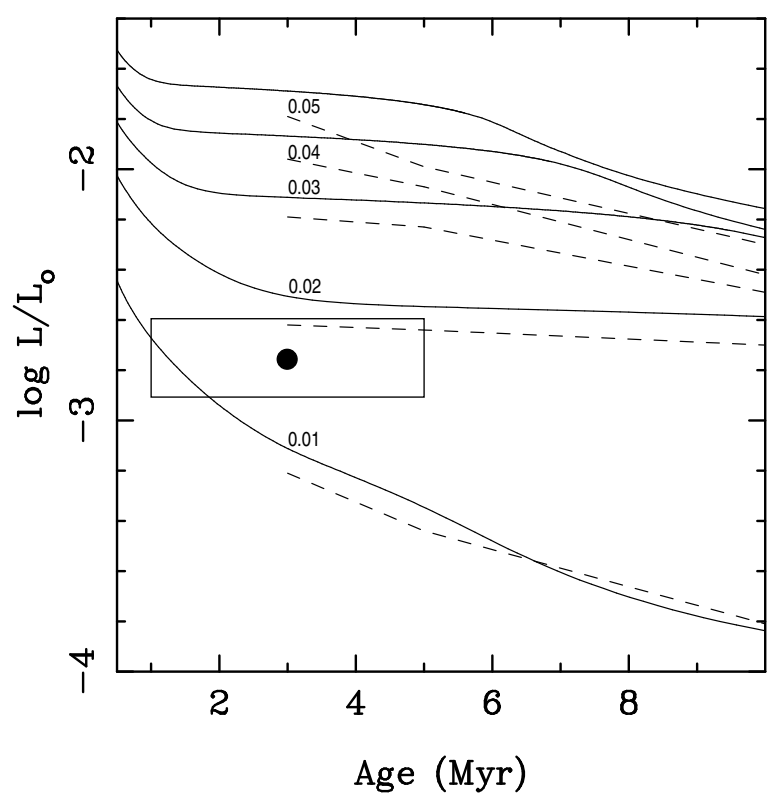

Fig. 3.- Time dependence of luminosity for very low-masses according to the theoretical models by Burrows et al. (1997, solid line) and the Lyon group (1999, dashed line). The region occupied by S Ori 47 considering the uncertainties in the determination of its luminosity and age is indicated by the box. Masses are labeled in solar units. 
TABLE 1

OBSERVED DATA AND BASIC PARAMETERS FOR S ORI 47.

\begin{tabular}{ccccccccccc}
\hline \hline$R-I^{\mathrm{a}}$ & $I-Z^{\mathrm{a}}$ & $I-J$ & $J_{\mathrm{UKIRT}}$ & $T_{\text {eff }}{ }^{\mathrm{b}}$ & $\log L / L_{\odot}$ & Sp. Type & H $\alpha$ & Li I $_{670.8}$ & Na I 819.5 & Cs I852.1 \\
\hline $2.4 \pm 0.3$ & $1.00 \pm 0.06$ & $3.3 \pm 0.1$ & $17.2 \pm 0.1$ & $2000 \pm 100 \mathrm{~K}$ & $-2.75 \pm 0.15$ & $\mathrm{~L} 1.5 \pm 0.5$ & $\leq 6^{\mathrm{c}}$ & $4.3 \pm 0.5^{\mathrm{c}}$ & $2.5 \pm 0.5^{\mathrm{c}}$ & $1.2 \pm 0.5^{\mathrm{c}}$ \\
\hline
\end{tabular}

${ }^{a}$ Colors taken from Béjar et al. (1999).

${ }^{\mathrm{b}}$ Assumes a dwarf spectral type conversion.

c Equivalent widths are given in $\AA$, wavelenghts in $\mathrm{nm}$. 